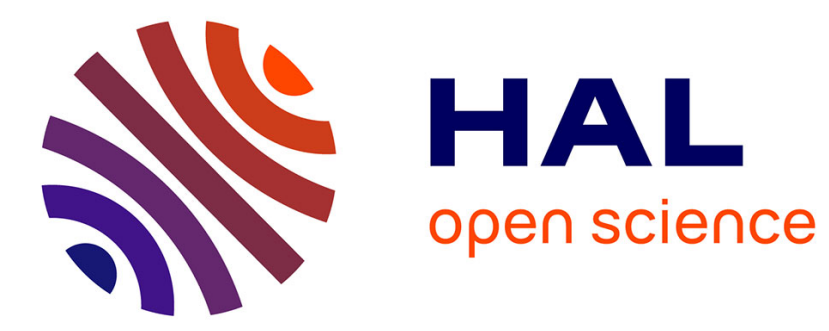

\title{
Drug-sponge lipid nanocarrier for in situ cargo loading and release using dynamic covalent chemistry
}

Fei Liu, Yosuke Niko, Redouane Bouchaala, Luc Mercier, Olivier Lefebvre, Bohdan Andreiuk, Thierry Vandamme, Jacky G Goetz, Nicolas Anton, Andrey Klymchenko

\section{To cite this version:}

Fei Liu, Yosuke Niko, Redouane Bouchaala, Luc Mercier, Olivier Lefebvre, et al.. Drug-sponge lipid nanocarrier for in situ cargo loading and release using dynamic covalent chemistry. Angewandte Chemie International Edition, In press, 10.1002/anie.202014259 . hal-03086687

\section{HAL Id: hal-03086687 https://hal.science/hal-03086687}

Submitted on 22 Dec 2020

HAL is a multi-disciplinary open access archive for the deposit and dissemination of scientific research documents, whether they are published or not. The documents may come from teaching and research institutions in France or abroad, or from public or private research centers.
L'archive ouverte pluridisciplinaire HAL, est destinée au dépôt et à la diffusion de documents scientifiques de niveau recherche, publiés ou non, émanant des établissements d'enseignement et de recherche français ou étrangers, des laboratoires publics ou privés. 


\title{
Drug-sponge lipid nanocarrier for in situ cargo loading and release using dynamic covalent chemistry
}

Fei Liu, ${ }^{1,2, \S}$ Yosuke Niko, ${ }^{1,3, \S}$ Redouane Bouchaala, ${ }^{1}$ Luc Mercier, ${ }^{4, \#}$ Olivier Lefebvre, ${ }^{4}$ Bohdan Andreiuk, ${ }^{1}$ Thierry Vandamme, ${ }^{2}$ Jacky G. Goetz, ${ }^{4}$ Nicolas Anton, ${ }^{2}$ Andrey Klymchenko ${ }^{1, *}$

${ }^{1}$ Université de Strasbourg, Laboratoire de Bioimagerie et Pathologies, UMR 7021 CNRS, 74 route du Rhin, 67401, Illkirch, France

${ }^{2}$ INSERM UMR 1260, Regenerative Nanomedicine (RNM), FMTS, CNRS 7199, CAMB, Université de Strasbourg, F-67000 Strasbourg, France

${ }^{3}$ Research and Education Faculty, Multidisciplinary Science Cluster, Interdisciplinary Science Unit, Kochi University, 2-5-1, Akebono-cho,Kochi-shi, Kochi, 780-8520, Japan

${ }^{4}$ Inserm U1109, Tumor Biomechanics, Fédération de Médecine Translationnelle de Strasbourg (FMTS), University of Strasbourg, F-67200, France

${ }^{\#}$ Current address: Interdisciplinary Institute for Neuroscience, University of Bordeaux, CNRS UMR 5297, 33077 Bordeaux, France

$\S$ These authors contributed equally to this work.

*Corresponding author: E-mail: andrey.klymchenko@unistra.fr

Supporting information for this article is given via a link at the end of the document.

\begin{abstract}
Currently, drug delivery strategies using nanocarriers (NCs) deal with encapsulation of cargo or its covalently modified prodrug. Here, we propose a concept of reversible $\mathrm{pH}$-controlled capture and delivery of active cargo based on dynamic covalent chemistry inside lipid nano-droplets (nanoemulsions), coined as "drug sponge". We designed a highly lipophilic hydrazide (LipoHD) capable to react with a free cargo-ketone (fluorescent dye and doxorubicin drug) directly inside lipid NCs, yielding lipophilic hydrazone prodrug efficiently captured in the oil core. LipoHD-loaded NCs spontaneously accumulated cargo-ketones, yielding formulations stable against cargo leakage at $\mathrm{pH}$ 7.4, and further released their dye/drug cargo at low $\mathrm{pH}$ range (5.0-6.8) in solution and live cells. Doxorubicin-loaded drug-sponge NCs showed cytotoxicity in four cancer cell lines and capacity to inhibit tumor growth in subcutaneous xenografts of mice. Finally, unprecedented extraction of dye/drug cargos directly from cells and tissues (i.e. detoxification) was realized by the drug-sponge NCs.
\end{abstract}




\section{Introduction}

In the last decades, nanoparticles (NPs) have been explored as nanocarriers (NCs) for the targeted delivery and controlled release of drug molecules. ${ }^{[1]}$ Current drug delivery systems based on NCs exploit two common paradigms, based on encapsulation (supramolecular) and covalent chemistry. ${ }^{\text {[2] }}$ In the first approach, the drug is non-covalently encapsulated inside the nanocarrier. ${ }^{[3]}$ The advantage of this method is that the cargo is not chemically modified before and after the release, however, noncovalent encapsulation is prone to burst release and poorly controlled leakage of the cargo. ${ }^{[4]}$ The second approach is based on covalent linkage of the drug inside the nanocarrier in form of a prodrug conjugate. ${ }^{[4 a, 5]}$ This method ensures high stability of the nanocarrier, while the use of cleavable bonds, such as acetals, orthoesters, hydrazones, disulfide, etc, enables stimuli-responsive cargo release under control of $\mathrm{pH}$, reduction, enzymes, etc. ${ }^{[6]}$ However, in this case, the drugs are modified chemically through a synthetic protocol, thus raising the question of solvent residues, toxicity and bio-distribution of corresponding prodrug. An ideal solution is to use NCs as a nano-reactor to form prodrug in situ, which after reaching the target cells can be cleaved, thus releasing the drugs under control of environment. In this case, the cargo enters and exits from the nanocarrier in its intact form, which would simplify the preparation and maximize the safety of the drug delivery.

New possibilities appear with dynamic covalent chemistry (DCvC), which deals with dynamic bonds that form and disrupt reversibly under environmental control. ${ }^{[7]} \mathrm{DCvC}$, which is at the interface of covalent and supramolecular chemistry, ${ }^{[8]}$ gave rise to new generation of materials with self-healing and adaptive properties. ${ }^{[9]}$ Reversible and environment-sensitive nature of the dynamic covalent bonds is particularly attractive for the field of drug delivery. ${ }^{[10]}$ The most popular dynamic covalent bonds include hydrazone, ${ }^{[11]}$ oxime, ${ }^{[12]}$ imine,${ }^{[13]}$ disulfide, ${ }^{[14]}$ boronate ester ${ }^{[15]}$ etc. On one hand, transient formation of these bonds enables crossing of cell membrane barriers for delivery of cargos inside the cells. ${ }^{[16]}$ On the other hand, these bonds can be used to build nanocarriers ${ }^{[10,13 b, 17]}$ and/or conjugate the nanocarrier with drugs ${ }^{[18]}$ in order to ensure efficient transport and controlled release of the cargo. ${ }^{[19]}$ Hydrazone bonds, sensitive to $\mathrm{pH}$, are particularly attractive in this respect, ${ }^{[11,20]}$ because targeted tumor tissues are characterized by low $\mathrm{pH} .{ }^{[19,21]}$ Moreover, after endocytosis of nanomedicines ${ }^{[22]}$ the lysosomal compartments can further contribute to $\mathrm{pH}$-controlled cleavage of the hydrazones. However, these reports were focused on the covalent approach, where the drug conjugates with the nanocarrier were synthesized before the formulation of the nanocarriers, whereas in situ loading of cargo into nanocarrier through dynamic covalent bonds has not been reported to date.

$\mathrm{DCvC}$ has been largely used for designing responsive nanocarriers based on polymers. ${ }^{\text {[10, }}$ 17, 20d] However, the polymer core is solid, and thus cannot serve as a nano-reactor for in situ conjugation of a cargo with a nanocarrier. Lipid-based NCs constitute a promising alternative to polymeric NPs in drug delivery applications. Traditionally, this field is dominated by liposomes, presenting lipid membrane and aqueous core, which has already been approved in clinics as pharmaceutical carriers. ${ }^{[23]}$ However, lipid membrane of liposomes is a good barrier for hydrophilic cargoes only, while encapsulation of hydrophobic cargoes (such as doxorubicin) requires their precipitation inside the liposome (as for Doxyl). Lipid nanoemulsions (NEs) present an oily core, which can serve as nano-reactor for encapsulation of drugs through $\mathrm{DCvC}$. NEs are highly promising biomimetic nanocarriers for drug delivery because they are (1) inexpensive and easy to produce; (2) composed of excipients generally recognized as safe (GRAS) and (3) biodegradable. ${ }^{[24]}$ Despite their liquid core, they exhibit rather long circulation time, preserve integrity of their core in the blood circulation and can accumulate in the tumor in nearly intact form. ${ }^{[25]}$ They were also shown to maintain their integrity after crossing a human intestinal epithelium barrier. ${ }^{[26]}$ However, a drug having medium lipophilicity has a tendency to leak out rapidly from lipid NCs into biological media, as shown for a model dye 
Nile Red in serum, ${ }^{[27]}$ whereas its modification with lipophilic chains can drastically inhibit the leakage. ${ }^{[27]}$ Moreover, recent studies showed that lipophilic prodrugs of doxorubicin bearing an alkyl chain improved anti-tumor potency of the drug-loaded nanoemulsions. ${ }^{[28]}$ Therefore, we hypothesized that lipid NCs (NEs) can be used as a nano-reactor to form lipophilic prodrugs in situ through DCvC. In this case, a specially designed lipophilic "capture" molecule could form dynamic covalent bond with a drug, i.e. forming prodrugs inside the nano-droplets, which would render the system stable against cargo leakage, and allow the stimuli-responsive drug release. Moreover, this "drug-sponge" system could capture in situ drug molecules from biological media and cells, which is an unexplored field in nanomedicine. Currently, active drug removal (or detoxification from drugs) is known in pharmacology and clinical practice, where excess of drug (e.g. an overdose) is removed by hemodialysis, plasma exchange and peritoneal dialysis. ${ }^{[29]}$ In order to ensure in situ drug capture in form of a prodrug, $\mathrm{DCvC}$ with a hydrazone bond can be considered. The latter has been frequently used in prodrugs design because it can be cleaved at weakly acidic $\mathrm{pH}(\sim 6)$ and readily applied to a drug bearing ketone/aldehyde groups like doxorubicin. ${ }^{[17 \mathrm{~d}, 20 \mathrm{c}, 30]}$

In the present study, we developed a concept of "drug-sponge", wherein a lipid NCs, encapsulating a lipophilic reactive capture molecule (LipoHD, Scheme 1), can spontaneously accumulate cargo by forming dynamic covalent bonds inside the oil core. The drug-sponge concept was validated in solution and live cells and further applied for drug release in tumor bearing mice and drug removal from tissues.
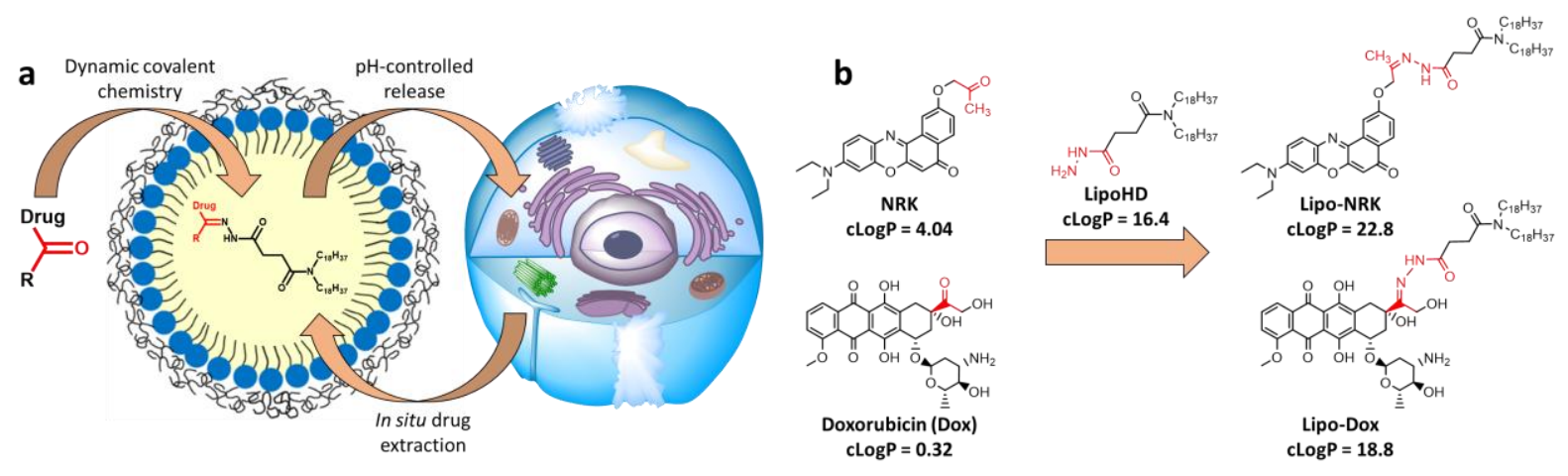

Scheme 1. Concept of drug-sponge nanocarrier (a) and chemical structures of cargoes (NRK and Dox), lipidic capture molecule (LipoHD) and corresponding pro-drug conjugates (b). Calculated $\log \mathrm{P}(\mathrm{cLog} \mathrm{P})$ are presented.

\section{Results and discussion}

To ensure efficient capture of cargo inside oil core of lipid NCs, we designed a highly lipophilic molecule (calculated $\log P, \operatorname{cog} P=16.4$ ) bearing hydrazide group (LipoHD, Scheme 1). It is expected to form dynamic covalent (hydrazone) bond with a cargo bearing aldehyde or ketone group. LipoHD was obtained in three steps starting from dioctadecylamine (Scheme S1). Two cargo-ketones were selected: Nile Red-ketone (NRK) as a model fluorescent cargo and doxorubicin (Dox) as an anti-cancer drug. NRK was synthesized by reacting Nile Red phenol with chloroacetone (Scheme S2). NRK and Dox, having medium lipophilicity (cLog P is 4.04 and 0.32, respectively), are expected to react with LipoHD inside oil core of NCs to form highly lipophilic "pro-drugs" Lipo-NRK and Lipo-Dox (cLog P is 22.8 and 18.8, respectively). This would ensure their retention inside NCs at 
neutral $\mathrm{pH}$ and cargo release at low $\mathrm{pH}$ after hydrazone bond hydrolysis. In addition, we also synthesized Lipo-NRK (Scheme S2) and Lipo-DOX (Scheme S3) conjugates in organic solvent and identified by NMR, Mass and FT-IR spectrometry.

We formulated NCs of around $80 \mathrm{~nm}$ size using nano-emulsification. ${ }^{[24 \mathrm{~b}]}$ A solution of presynthesized Lipo-NRK (10 mM) or control dye NRK $(10 \mathrm{mM})$ in Labrafac oil was mixed with Kolliphor ELP® surfactant and then formulated in milliQ water, yielding desired NCs. To study the NRK cargo release, we co-encapsulated lipophilic blue dye F888 ${ }^{[27]}$ that serves as energy donor for NRK in the Förster Resonance Energy Transfer (FRET) ${ }^{[25,27,31]}$ assay (Fig. 1a). NRK and Lipo-NRK NCs showed similar absorbance values at the maximum $~ 530 \mathrm{~nm}$ (Fig. S1a), indicating that encapsulation was successful in both cases. The FRET NCs excited at $405 \mathrm{~nm}$ (donor) showed both donor and acceptor emission bands (Fig. S1b), confirming FRET inside NCs between F888 donor and acceptor (NRK or Lipo-NRK).

Then, we mixed our FRET NCs with blank NCs, which are expected to work as a recipient medium for the hydrophobic cargo. The presence of blank NCs led to nearly complete loss of FRET for NRK/F888 NCs: the NRK fluorescence intensity dropped while emission of F888 increased (Fig. S1c). This result suggested the release of NRK and its transfer to blank NCs (Fig. 1a). In sharp contrast, fluorescence spectrum of FRET NCs with Lipo-NRK was only marginally affected, suggesting that Lipo-NRK remains inside the NCs without significant release. It should be also noted that after two weeks, the NRK formulation showed signs of dye precipitation, in line with our earlier data for parent Nile Red, ${ }^{[27]}$ whereas the corresponding Lipo-NRK NCs were stable.

As the hydrazone bond is cleavable at low $\mathrm{pH}$, we incubated Lipo-NRK/F888 NCs with 10-fold excess of blank NCs at pH varied from $\mathrm{pH} 7.4$ to $\mathrm{pH} 5$ (Fig. 1 and S2). For $\mathrm{pH}$ 7.4, the emission spectra remained stable over $8 \mathrm{~h}$, suggesting no dye release (Fig. 1b). By contrast, at $\mathrm{pH} 6.8$, the donor band gradually increased, while the acceptor band decreased over time (Fig. S2). This trend became more pronounced at lower pH (Fig. 1c and S2). The plotted acceptor/donor ratio vs time (Fig. 1d) showed a clear time-dependent release of NRK in $\mathrm{pH}$ range 5.0-6.8 and practically no release at neutral $\mathrm{pH}$.
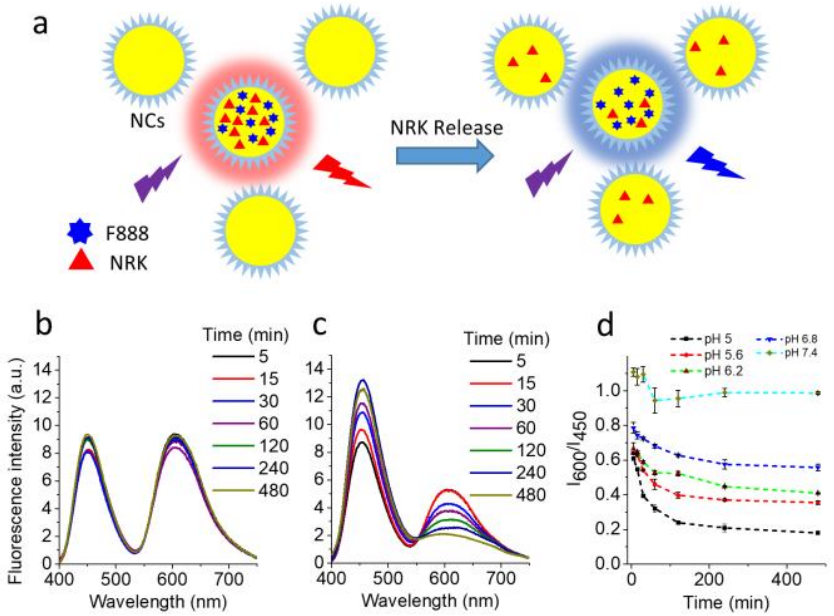

Fig. 1. (a) Schematic presentation of changes in FRET occurring during the cargo release. (b,c) Fluorescence spectra of Lipo-NRK NCs for different incubation time at $\mathrm{pH} 7.4$ (b) and $\mathrm{pH} 5$ (c) after dilution (1000-fold) in the presence of 20-fold excess of blank NCs. (d) Fluorescence intensity ratio of acceptor $\left(\mathrm{I}_{600}\right)$ to donor $\left(\mathrm{I}_{450}\right)$ over time at different $\mathrm{pH}$. Excitation wavelength was $405 \mathrm{~nm}$.

Our next question was whether prodrug model Lipo-NRK can be generated in situ inside NCs without synthesis of the "prodrug" in a flask (Fig. 2a). To this end, NCs containing LipoHD were 
first formulated and then incubated for $6 \mathrm{~h}(\mathrm{rt})$ with $\mathrm{NRK}$ in $\mathrm{pH} 4$. The reaction mixture then was then extracted by dichloromethane and studied by TLC. Lipo-NRK could be clearly identified as a new spot with higher Rf, which corresponded to pre-synthesized conjugate Lipo-NRK (Fig. 2b). Mass spectrometry of the dichloromethane extract confirmed the presence of Lipo-NRK (Fig. S3), confirming that the hydrazone conjugate can form in situ. To monitor formation of the conjugate in situ inside NCs, we prepared NCs encapsulating F888 as a FRET donor for NRK. NCs containing LipoHD showed increase in the FRET signal (Fig. 2c) as a growth of NRK emission intensity (at 600 $\mathrm{nm}$ ) and a decrease in F888 intensity (at $450 \mathrm{~nm}$ ), whereas no change was observed for NCs without LipoHD (Fig. 2d). These results suggested that Lipo-NRK conjugate was formed in the NCs containing LipoHD. Monitoring acceptor/donor ratio vs time revealed relatively fast hydrazone formation within first $30 \mathrm{~min}$ (Fig. 2e).
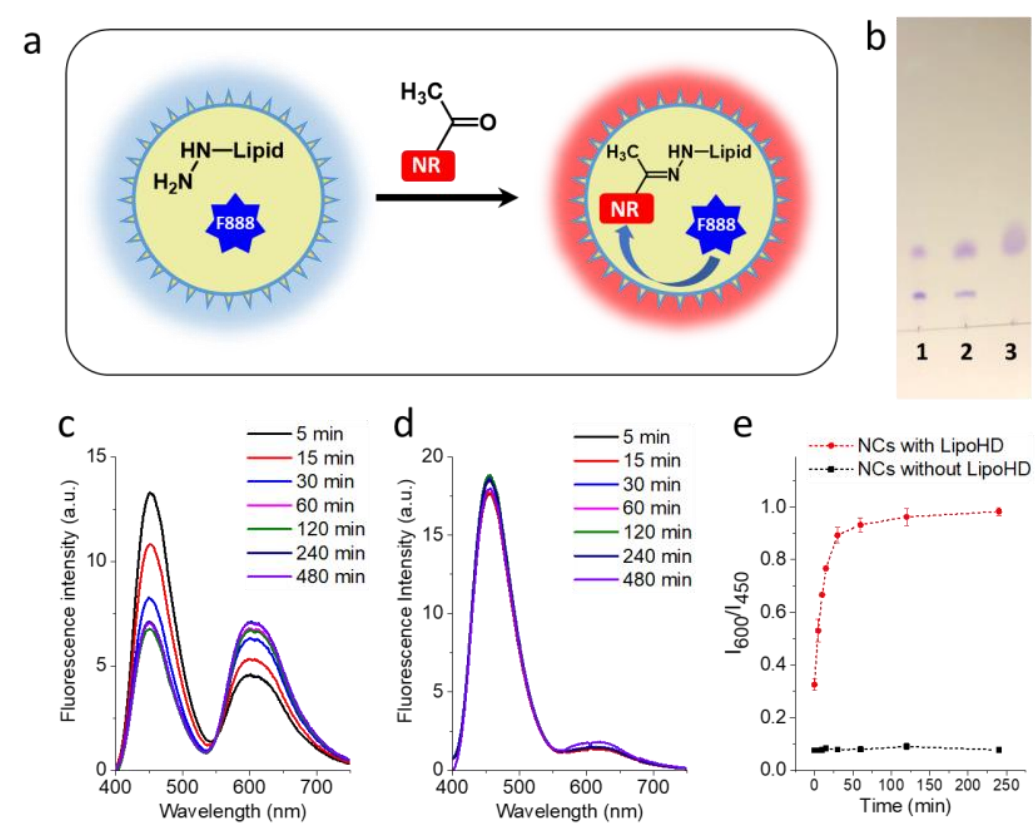

Fig. 2. (a) Scheme of in situ reaction of NRK and LipoHD monitored by FRET using F888 as energy donor. (b) TLC evidence of in situ formation of Lipo-NRK conjugate inside LipoHD NCs: (1) extracted reaction mixture of LipoHD NCs with NRK in DCM phase; (2) the same extracted mixture with pure Lipo-NRK; (3) pure Lipo-NRK. (c) Fluorescence spectra of the reaction mixture of LipoHD/F888 NCs with NRK at different reaction times after dilution (1000-fold) with 20-fold excess of blank NCs. Excitation wavelength is $405 \mathrm{~nm}$. (d) The same experiments as (c) for NCs without LipoHD. (e) Fluorescence intensity ratio at fixed wavelengths (600 to $450 \mathrm{~nm}$ ) for experiments (c) and (d). The in situ reaction was done at $\mathrm{pH} 4$.

Next, we evaluated possibility to form in situ prodrug of doxorubicin (Lipo-Dox) inside NCs using LipoHD. Dox is poorly soluble in oil ( $\sim 0.001 \mathrm{wt} \%$ in Labrafac), making inefficient its encapsulation into lipid NCs. By contrast, lipophilic hydrazone conjugate Lipo-Dox, pre-synthesized in organic solvent, showed high solubility in Labrafac oil (>1 wt \%). Blank and LipoHD-loaded NCs were incubated with Dox for $24 \mathrm{~h}$ at $\mathrm{pH}$ 4. After the dialysis, no absorbance of Dox was detected in control NCs without LipoHD, whereas the absorbance of Dox was significant for LipoHD NCs (Fig. S4). The result was also confirmed by visual observation of aqueous phase after dialysis (Fig. S4a). Dox encapsulation with respect to the mass of Labrafac oil was $0.48 \%$ based on extinction coefficient of Dox $\left(12,200 \mathrm{M}^{-1} \mathrm{~cm}^{-1}\right) .{ }^{[32]}$ Thus, LipoHD makes Dox prone to accumulation inside of NCs. The encapsulation efficiency of Dox into LipoHD-loaded NCs, calculated based on the absorbance of DOX before and after dialysis (Fig. S4b) was 25.8\%, whereas for control NCs without LipoHD it was close to zero. 
In order to monitor the in situ formation of Dox prodrug within NCs, a FRET system for Dox was designed. As a FRET couple of F888 with Dox gave too weak FRET signal (Fig. S5), we selected a far-red lipophilic Cy5 dye (DiD), already used as FRET acceptor for Dox. ${ }^{[33]}$ NCs loaded with DiD and LipoHD were incubated with Dox. The emission spectra of NCs excited at Dox absorption wavelength $(490 \mathrm{~nm})$ revealed the gradual increase in the FRET acceptor emission over time with kinetics (the acceptor/donor intensity ratio vs time) on the time scale of hours, whereas NCs without LipoHD showed practically no ratio change (Fig. S6). According to Dox absorption spectra (Fig. S7a), the encapsulation efficiency of Dox into LipoHD NCs was $28.6 \%$, and resulted loading of Dox was $0.52 \%$ in oil (w/w), consistent with data for LipoHD NCs without DiD (Fig. S4b). Thus, our results showed that a drug (or a dye) can be efficiently loaded inside lipid nanocarrier through in situ dynamic covalent bond formation generating encapsulated prodrug. To the best of our knowledge, such "drug-sponge" in situ drug loading has not been reported to date.

We further exploited the dynamic nature of the hydrazone bond to trigger $\mathrm{pH}$-controlled release of Dox from NCs (Fig. 3, S8), similarly to experiments with NRK. At pH 7.4, minimal changes of the FRET spectra (Fig. 3a) and the acceptor/donor ratio (Fig. 3f) were observed over $8 \mathrm{~h}$, suggesting relatively slow release of Dox at $\mathrm{pH}$ 7.4. By contrast, at $\mathrm{pH}$ 6.8, gradual loss of FRET was observed and this trend was stronger with gradual decrease of $\mathrm{pH}$ (Fig. 3b-f). We also found that the Lipo-Dox NCs were stable in physiological buffer PBS (pH 7.4) and in $10 \%$ serum (Fig. S8). Thus, with help of LipoHD, Dox release can be triggered by weakly acidic pH (5.0-6.8), which is important for delivery of drugs to acidic microenvironment of cancer tissues. ${ }^{[19,21]}$

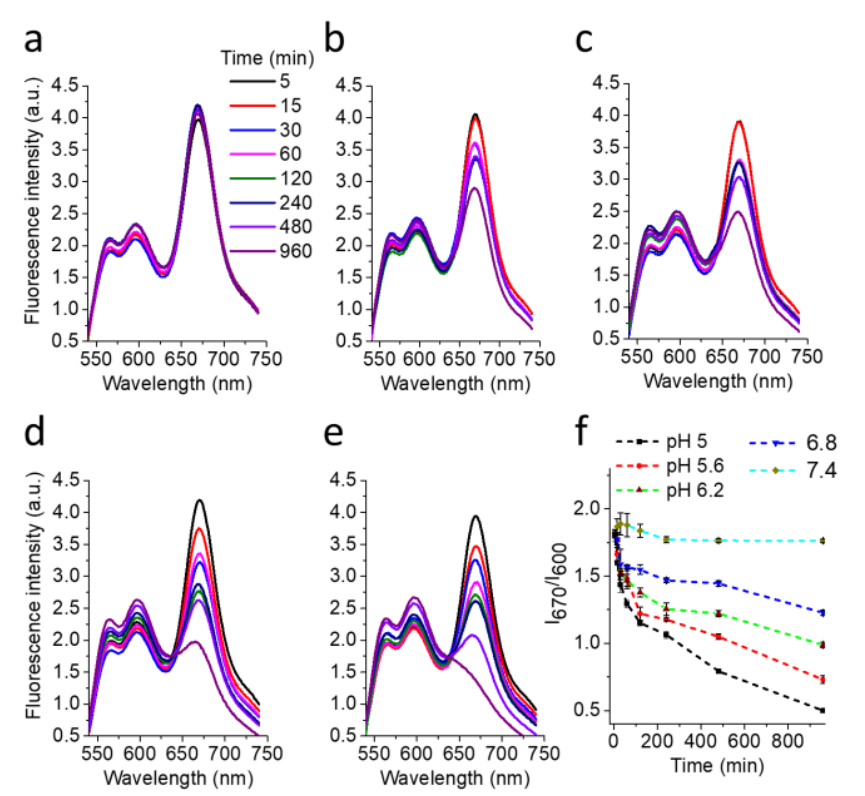

Fig. 3. Drug (Dox) release from NCs under $\mathrm{pH}$ control, studied by FRET from Dox to Cy5 dye (DiD). (a-e) Fluorescence spectra of Lipo-Dox FRET NCs (1000-fold dilution) in the presence of 20-fold excess of blank NCs at pH 7.4 (a), pH 6.8 (b), pH 6.2 (c), pH 5.6 (d) and pH 5 (e) over time. Excitation wavelength was $520 \mathrm{~nm}$. (f) Fluorescence intensity ratio of acceptor to donor (670 to $600 \mathrm{~nm}$ ) at different $\mathrm{pH}$ over time.

We compared sizes and polydispersity of different formulations used in this study. The size of LipoHD NCs were close to blank NCs (78 and $85 \mathrm{~nm}$ respectively), which implies that it does not influence directly the NCs formulation (Table S1). NRK formulations without LipoHD showed 
unexpectedly large size (112 nm). By contrast, NRK conjugate with LipoHD favored small and uniform NCs $(71 \mathrm{~nm})$. In case of Dox formulations, the samples after dialysis showed systematically lower size $(70-85 \mathrm{~nm})$ and polydispersity compared to those before dialysis $(105-144 \mathrm{~nm})$. Importantly, the Dox-LipoHD NCs size was close to that of blank NCs, which means that drug loading did not affect much the particles.

To study the $\mathrm{pH}$-dependent release of cargo in cells, NCs loaded with dye Lipo-NRK or prod-drug Lipo-Dox were incubated with HeLa cells at pH 7.4 and 5 of PBS followed by washing. At pH 7.4, Lipo-NRK NCs and Lipo-Dox NCs showed poor fluorescence inside the cells for both 1 and $30 \mathrm{~min}$ incubation (Fig. 4). By contrast, at pH 5 the release of both NRK and Dox was observed after $30 \mathrm{~min}$. Thus, at neutral pH the Lipo-NRK NCs and Lipo-Dox NCs are relatively stable in the presence of cells, but undergo release of NRK and Dox, respectively, at low $\mathrm{pH}$ because of hydrazone hydrolysis. However, the release of Dox was somewhat faster compared to NRK, which can be explained by lower lipophilicity of Lipo-Dox compared to Lipo-NRK (cLogP is 18.8 vs 22.8) that favors higher access to water of encapsulated Lipo-Dox and thus faster hydrazone hydrolysis, in line with our FRET studies. Control experiments with free NRK and Dox at both $\mathrm{pH} 7.4$ and $\mathrm{pH} 5$ showed some signal already after $1 \mathrm{~min}$ and strong emission after $30 \mathrm{~min}$ incubation (Fig. 4), suggesting rapid accumulation of the free cargos in cells independently of $\mathrm{pH}$.
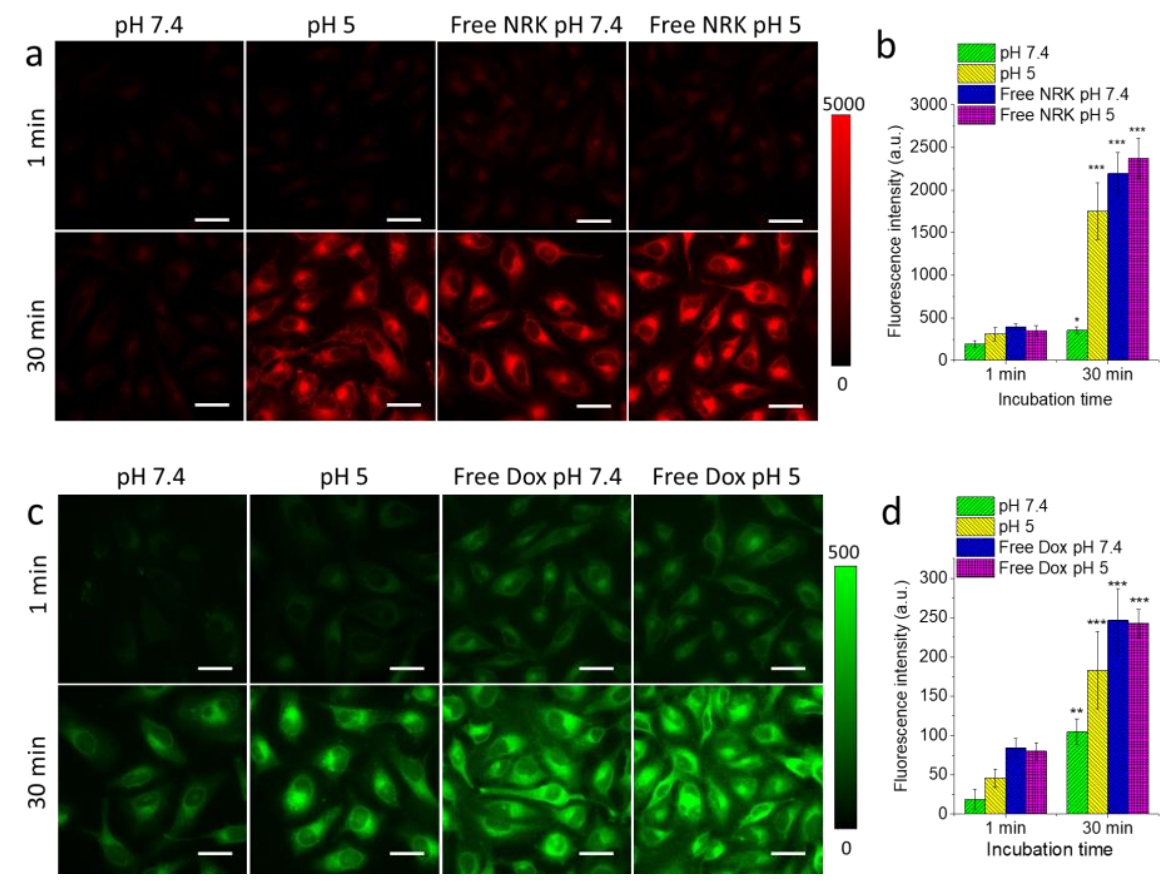

Fig. 4. pH-controlled release of cargos from NCs into cells, studied by fluorescence microscopy. (a) Fluorescence images of HeLa cells incubated for different time with Lipo-NRK NCs or free NRK at pH 5 and 7.4 (PBS). Excitation wavelength was $550 \mathrm{~nm}$ and the emission filter was $600 \mathrm{~nm}$. (b) Mean fluorescence intensity measured in 13 cells from (a) excluding the nucleus area. (c) Fluorescence images of HeLa cells incubated for different time with Lipo-Dox NCs or free Dox at pH 5 and 7.4 (PBS). Excitation wavelength was $470 \mathrm{~nm}$ and the emission filter was $530 \mathrm{~nm}$. Scale bars (a,c): 40 $\mu \mathrm{m}$. (d) Mean fluorescence intensity measured in 13 cells from (c) excluding the nucleus area. Statistical analysis (vs. data at pH 7.4, $1 \mathrm{~min})$ : $\mathrm{p}<0.5(*), \mathrm{p}<0.1(* *), \mathrm{p}<0.01(* * *)$.

It remained unclear whether NRK and Dox cargos are liberated from NCs inside the cells or in the extracellular medium. The absence of signal for Lipo-NRK NCs in cells after $30 \mathrm{~min}$ (Fig. 4a) implies 
that NCs did not rapidly internalize inside the cells. This stealth property, also observed earlier for lipid NCs loaded with lipophilic dye NR688, ${ }^{[27,34]}$ is probably linked to their PEG shell. To further verify accumulation of NCs inside the cells, we co-encapsulated our cargos with corresponding lipophilic dye: F888 with NRK and DiD with Dox. After 30 min incubation, neither NRK nor Dox formulations showed signal of corresponding F888 and DiD markers (Fig. S9), suggesting absence of NCs inside the cells. By contrast, NRK and Dox channels showed strong signal of the corresponding cargo for LipoHD-based NCs at $\mathrm{pH} 5$ and for control NCs at pH 7.4 (Fig. S9), in line with the data above. We can conclude that at low pH Lipo-NRK NCs and Lipo-Dox NCs release their cargo into extracellular medium followed by cargo internalization inside cells.

Since our Lipo-Dox NCs can slowly release Dox in vitro, we studied cytotoxicity of Lipo-Dox NCs with that of free Dox in Hela cells after 12, 24 and 48h incubation in culture medium (DMEM). For both formulations, clear cytotoxicity was observed for concentration $\geq 5 \mu \mathrm{g} / \mathrm{mL}$ and it increased with the incubation time (Fig. S10). Generally, Lipo-Dox NCs showed a slightly lower cytotoxicity than free Dox, especially for shorter incubation times. This difference in cytotoxicity is probably related to the delayed release of Dox from Lipo-Dox NCs. Nevertheless, after 48h Lipo-Dox NCs showed high toxicity, similarly to free Dox, suggesting that this incubation is sufficiently long to release free Dox from NCs. Furthermore, we studied cytotoxicity of the two formulations in three mammary carcinoma cell lines: 4T1, D2A1 from mouse and MDA-MB-231 from human after incubation for 48 hours. Both free Dox and Lipo-Dox NCs were found toxic, with cell viability <50\% for concentration $\geq 5 \mu \mathrm{g} / \mathrm{mL}$ (Fig. S11), although some small differences were observed dependent on a cell line.

Then, the in vivo therapeutic potential of Lipo-Dox NCs was investigated on mice with xenograft tumors (D2A1 and MDA-MB-231, Fig. S12). We observed reduced tumor growth profiles in mice that were administered with Lipo-Dox NCs, when compared to control administered with only NCs, which is especially pronounced for D2A1 (Fig. S12b). Moreover, in case of D2A1 cells, Lipo-Dox NCs showed more effective suppression of tumor growth, compared to free Dox, which can be explained two factors. First, Dox is poorly soluble in water and thus can aggregate, in contrast to Lipo-Dox formulation. Moreover, we previously showed that lipid NCs of $\sim 100 \mathrm{~nm}$ diameter exhibit strong EPR in D2A1 xenografted tumors, ${ }^{[25]}$ which can favor accumulation of Lipo-Dox NCs in tumor areas. A dedicated in vivo study will be needed to explore full therapeutic potential of Lipo-Dox system.

Inspired by a capacity of LipoHD NCs to capture molecules in situ through dynamic covalent bond formation, we wondered whether these NCs can extract ketones, like NRK and Dox, from cells. The cells were treated with NRK or Dox and then incubated in LipoHD NCs, blank NCs and control PBS for 1 and $60 \mathrm{~min}$. In PBS, the changes in the fluorescence were negligible for both NRK and Dox, indicating that they remained inside the cells (Fig. 5). In case of blank NCs some decrease in the fluorescence intensity was observed, which could be explained by partial extraction of these apolar molecules into the hydrophobic reservoirs of NCs. In case of Dox, this non-specific extraction was much less efficient, probably because Dox is less lipophilic than NRK (corresponding cLogP is 0.32 vs 4.04). Strikingly, LipoHD NCs drastically decreased the fluorescence intensity of both NRK and Dox after 60 min incubation, which was much stronger compared to blank NCs (Fig. 5). Then, we characterized by fluorescence spectroscopy the extraction media (LipoHD NCs, blank NCs and PBS) incubated with HeLa cells for different time. Fluorescence intensity increased over the studied period of $1 \mathrm{~h}$ for all three media, whereas LipoHD NCs showed the strongest intensity increase (Fig. S13). Using a calibration curve of the peak fluorescence intensity vs NRK concentration (Fig. S13), we found that the amount of extracted NRK after $1 \mathrm{~h}$ was $0.41,0.29$ and 0.14 pmol for LipoHD NCs, blank NCs and PBS, respectively. Given that that the estimated total amount of NRK uptaken by cells 
(see details in SI) was $0.5 \mathrm{pmol}$, the three media extracted respectively 82,58 and $28 \%$ of NRK form cells. This result confirmed our microscopy data showing that LipoHD NCs are significantly more effective extraction agent than the control media. Importantly, TLC and mass spectrometry of the LipoHD NCs extract from cells revealed the presence of LipoHD-NRK conjugate (Fig. S14). Thus, we can conclude that this much higher efficiency of extraction with LipoHD NCs is connected with the formation of the dynamic covalent bonds of ketone molecules (NRK and Dox) with the "drug sponge" NCs. As mentioned above, within this short time period NCs cannot enter the cells, so that they extract the internalized ketones being outside the cells. Lipophilic NRK and Dox molecules probably diffuse freely within the lipophilic compartments of the cells, and therefore can reversibly reach the plasma membranes, where they can be transferred to LipoHD NCs. This extraction process is reverse to a classical accumulation of lipophilic molecules in cells by passive diffusion and it is energetically favorable here because of formation of dynamic covalent bonds between the lipophilic ketones and Lipo-HD inside NCs. The obtained result provides the first proof of concept for the use of $\mathrm{DCvC}$ for removing drug molecules from cells.
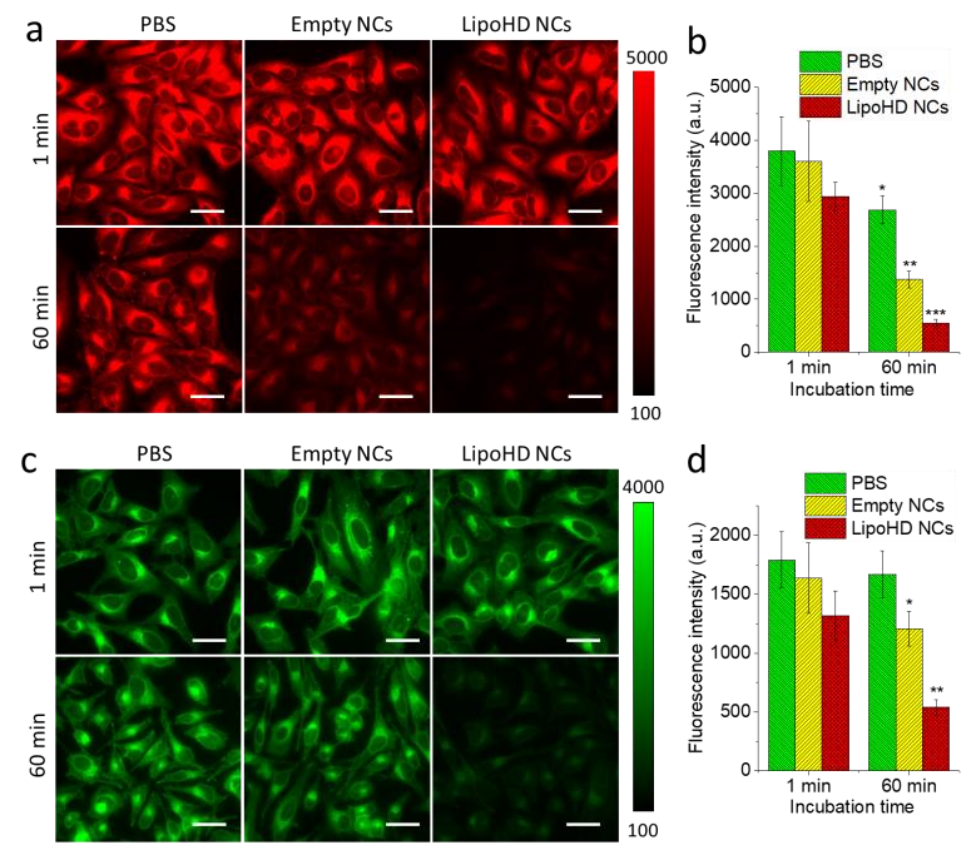

Fig. 5. In situ extraction of ketone drugs by LipoHD NCs from HeLa cells. (a) In situ extraction of NRK: cells were pre-incubated with $1 \mu \mathrm{M}$ of NRK for $30 \mathrm{~min}$, washed and then treated for 1 or 60 min with 100-times diluted LipoHD NCs, blank NCs and PBS and then washed with PBS before imaging. Excitation wavelength was $550 \mathrm{~nm}$ and the emission filter was $600 \mathrm{~nm}$. (b) Mean fluorescence intensity measured in 15 cells from (a) excluding the nucleus area. (c) In situ extraction of Dox: cells were pre-incubated with $15 \mu \mathrm{M}$ of Dox for $30 \mathrm{~min}$, washed, treated or 1 or 60 min with 100-times diluted LipoHD NCs, blank NCs and PBS and then washed with PBS before imaging. Excitation wavelength was $470 \mathrm{~nm}$ and the emission filter was $530 \mathrm{~nm}$. Scale bars (a,c): $40 \mu \mathrm{m}$. (d) Mean fluorescence intensity measured in 15 cells from (c) excluding the nucleus area. Statistical analysis (vs. data in PBS, 1 min): $\mathrm{p}<0.5(*), \mathrm{p}<0.1(* *), \mathrm{p}<0.01(* * *)$. 
We further explored the capacity of LipoHD NCs to extract ketones from tissues. To this end we selected chicken skin presenting lipid-rich adipose tissue, ${ }^{[35]}$ which is expected to uptake lipophilic molecules/drugs such as NRK and Dox. Transmission image of the skin showed characteristic morphological features of adipose tissue with individual cells (Fig. 6). However, only faint autofluorescence was observed in chicken skin (Fig. S15). Incubation of the skin with NRK or Dox in PBS resulted in a strong fluorescence signal from the tissue (Fig. 6 and S16), suggesting their accumulation in the tissue. Right after addition of blank NCs or LipoHD NCs to the stained chicken skin, the fluorescence signal remained close to that in PBS (Fig. 6 and S16) treated samples. However, after incubation for $30 \mathrm{~min}$, the fluorescence intensity for LipoHD NCs dropped significantly for both NRK and Dox $(\mathrm{p}<0.01)$, whereas blank NEs showed much smaller $(\mathrm{p}<0.5)$ decrease and PBS did not show any change for NRK and Dox (Fig. 6 and S16). Thus, LipoHD NCs are able to extract ketones, including Dox drug, highlighting the potential of our drug-sponge NCs as a detoxification platform.

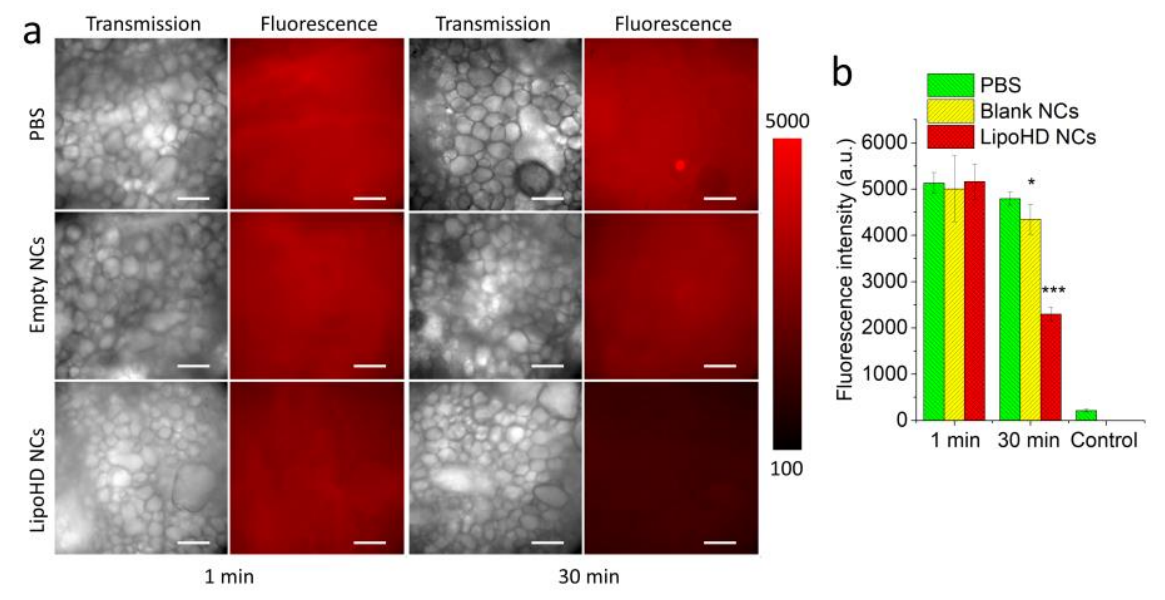

Fig. 6. In situ extraction of ketone drug Dox by LipoHD NCs from chicken skin adipose tissue. (a) Transmission and fluorescence images of in situ extraction of Dox: the skin tissue was pre-incubated with $45 \mu \mathrm{M}$ of Dox for 10 min and treated for 1 or 30 min with 100-fold diluted LipoHD NCs, blank NCs or PBS. Excitation wavelength was $550 \mathrm{~nm}$ and the emission filter was $600 \mathrm{~nm}$. Scale bar: 120 $\mu \mathrm{m}$. (b) Mean fluorescence intensity measured in the center area (3 images analyzed per condition). Statistical analysis (vs. data in PBS, $1 \mathrm{~min})$ : $\mathrm{p}<0.5(*), \mathrm{p}<0.01(* * *)$.

\section{Conclusions}

In this work, we propose a concept of reversible $\mathrm{pH}$-controlled delivery of active cargo (drug or contrast agent) to the cells based on $\mathrm{DCvC}$ inside lipid nano-droplets, so-called "drug sponge". As most of drugs cannot be kept inside oil core of the lipid nano-droplets because of their moderate lipophilicity, they need to be modified with a highly lipophilic moiety to obtain a lipophilic prodrug conjugate. Here, we changed the prodrug strategy and propose to generate the conjugate directly inside the oil core of a nanocarrier by a dynamic covalent bond between a lipophilic molecule and the cargo. We designed a highly lipophilic hydrazide (LipoHD) capable of forming a hydrazone bond with a cargo (fluorescent dye and doxorubicin drug) bearing ketone group. Lipid nanoemulsions loaded with LipoHD were able to spontaneously accumulate cargo-ketones with a kinetics on the time scale of hours. In contrast to simple formulations of nanocarriers with cargos, nanocarriers loaded using dynamic covalent chemistry were stable at $\mathrm{pH} 7.4$ against cargo leakage. At low pH (56.8), relevant to endosomes and cancer tissues, the cargos were successfully released, because of $\mathrm{pH}$ dependent hydrolysis of the hydrazone. This drug-sponge system was validated in a cancer cell line, 
where it showed efficient $\mathrm{pH}$-controlled release of dye/drug cargos into the cells. LipoHD NCs loaded with doxorubicin showed cytotoxicity in four cell lines after $48 \mathrm{~h}$ incubation, similarly to free doxorubicin. The therapeutic potential of drug-sponge NCs loaded with doxorubicin was demonstrated for xenografted tumors (D2A1 and MDA cells) on mice. Remarkably, the capacity of our drug sponge to capture cargo molecules in situ was successfully applied to extract fluorescent ketone and doxorubicin directly from cancer cells as well as from chicken skin adipose tissue. Thus, in addition to conventional $\mathrm{pH}$ controlled release, our drug sponge can extract the drug from cells and lipid-rich tissues (e.g. chicken skin), which is an unprecedented result for nanoparticles. The unique features of our drug-sponge NCs rely on liquid nature of the oil core, which allows in situ reactions, and the use of dynamic covalent bonds. In contrast to conventional covalent approach where prodrug is pre-synthesized, ${ }^{[4 a, 5]} \mathrm{DCvC}$ ensures that chemically stable capture molecule (LipoHD) can react in situ with cargo forming a prodrug directly inside the nanocarrier and, then, at low $\mathrm{pH}$ and high dilution, the reaction is reversed towards the cargo release. Remarkably, in this cargo loading-release cycle, prodrug is expected to remain inside the oil core, so that originally loaded cargo is released to biological subject in its intact form. In this way, the approach is different from conventional prodrug approach, where pre-synthesized prodrug is brought directly in contact with cells and tissues. We expect that the developed drug-sponge concept can be applied to any drug/contrast agent bearing ketone or aldehyde group. Moreover, the concept could be extended to other dynamic covalent bonds, which can enlarge the scope of cargos. Finally, the capacity of the drug sponge nanocarriers to extract drugs from the cells and adipose tissue of chicken skin opens new possibilities in detoxification from toxins and excess of used drugs.

\section{Acknowledgements}

This work was supported by the European Research Council ERC Consolidator grant BrightSens 648528, SATT Conectus, institutional funds from INSERM, CNRS and University of Strasbourg. FL is supported from China Scholarship council (CSC). Y.N. was supported by a JSPS Fellowship for Research Abroad. B.A. was supported by LabEx Chimie des Systèmes Complexes. L.M was supported by INSERM and Region Alsace and by SATT Conectus. We thank Valentin Gensbittel for assistance with some drug removal experiments.

\section{Conflict of interest}

European patent application $\mathrm{n}^{\circ} 19305035.8$ has been deposited.

Keywords: controlled release; dynamic covalent bonds; fluorescence; nanotechnology; prodrugs.

\section{References}

[1] T. M. Allen, P. R. Cullis, Science 2004, 303, 1818.

[2] a) M. J. Webber, R. Langer, Chem. Soc. Rev. 2017, 46, 6600; b) H. Cabral, K. Miyata, K. Osada, K. Kataoka, Chem. Rev. 2018, 118, 6844.

[3] A. S. Narang, D. Delmarre, D. Gao, Int. J. Pharm. 2007, 345, 9.

[4] a) S. Mura, D. T. Bui, P. Couvreur, J. Nicolas, J. Control. Release 2015, 208, 25; b) C. R. de Azevedo, M. von Stosch, M. S. Costa, A. M. Ramos, M. M. Cardoso, F. Danhier, V. Preat, R. Oliveira, Int. J. Pharm. 2017, 532, 229.

[5] a) V. Delplace, P. Couvreur, J. Nicolas, Polym. Chem. 2014, 5, 1529; b) D. Irby, C. Du, F. Li, Mol. Pharm. 2017, 14, 1325.

[6] a) W. W. Gao, J. M. Chan, O. C. Farokhzad, Mol. Pharm. 2010, 7, 1913; b) F. Seidi, R. Jenjob, D. Crespy, Chem. Rev. 2018, 118, 3965; c) M. Karimi, et al., Chem. Soc. Rev. 2016, 45, 1457. 
[7] a) A. Herrmann, Chem. Soc. Rev. 2014, 43, 1899; b) Y. H. Jin, C. Yu, R. J. Denman, W. Zhang, Chem. Soc. Rev. 2013, 42, 6634; c) S. J. Rowan, S. J. Cantrill, G. R. L. Cousins, J. K. M. Sanders, J. F. Stoddart, Angew. Chem.-Int. Edit. 2002, 41, 898.

[8] J. M. Lehn, Chem. Soc. Rev. 2007, 36, 151.

[9] a) J. M. Lehn, Angew. Chem.-Int. Edit. 2015, 54, 3276; b) Y. Zhang, M. Barboiu, Chem. Rev. 2016, 116, 809; c) N. Roy, B. Bruchmann, J. M. Lehn, Chem. Soc. Rev. 2015, 44, 3786.

[10] S. Ulrich, Acc. Chem. Res. 2019, 52, 510.

[11] S. J. Sonawane, R. S. Kalhapure, T. Govender, Eur. J. Pharm. Sci. 2017, 99, 45.

[12] D. K. Kolmel, E. T. Kool, Chem. Rev. 2017, 117, 10358.

[13] a) A. Dizdarevic, N. A. Efiana, T. N. Q. Phan, B. Matuszczak, A. Bernkop-Schnurch, Eur. J. Pharm. Biopharm. 2019, 142, 92; b) C. A. Zentner, F. Anson, S. Thayumanavan, T. M. Swager, J. Am. Chem. Soc. 2019, 141, 18048.

[14] S. P. Black, J. K. M. Sanders, A. R. Stefankiewicz, Chem. Soc. Rev. 2014, 43, 1861.

[15] B. Akgun, D. G. Hall, Angew. Chem.-Int. Edit. 2016, 55, 3909.

[16] a) P. R. Westmark, S. J. Gardiner, B. D. Smith, J. Am. Chem. Soc. 1996, 118, 11093; b) X. Wu, N. Busschaert, N. J. Wells, Y. B. Jiang, P. A. Gale, J. Am. Chem. Soc. 2015, 137, 1476; c) E. K. Bang, G. Gasparini, G. Molinard, A. Roux, N. Sakai, S. Matile, J. Am. Chem. Soc. 2013, 135, 2088; d) G. Gasparini, G. Sargsyan, E. K. Bang, N. Sakai, S. Matile, Angew. Chem.-Int. Edit. 2015, 54, 7328.

[17] a) J. M. Priegue, D. N. Crisan, J. Martinez-Costas, J. R. Granja, F. Fernandez-Trillo, J. Montenegro, Angew. Chem.-Int. Edit. 2016, 55, 7492; b) J. M. Priegue, I. Lostale-Seijo, D. Crisan, J. R. Granja, F. Fernandez-Trillo, J. Montenegro, Biomacromolecules 2018, 19, 2638; c) V. S. Vyas, M. Vishwakarma, I. Moudrakovski, F. Haase, G. Savasci, C. Ochsenfeld, J. P. Spatz, B. V. Lotsch, Adv. Mater. 2016, 28, 8749; d) M. Prabaharan, J. J. Grailer, S. Pilla, D. A. Steeber, S. Q. Gong, Biomaterials 2009, 30, 5757.

[18] C. Z. Ding, Z. B. Li, Mater. Sci. Eng. C-Mater. Biol. Appl. 2017, 76, 1440.

[19] M. Kanamala, W. R. Wilson, M. M. Yang, B. D. Palmer, Z. M. Wu, Biomaterials 2016, 85, 152.

[20] a) T. Etrych, L. Kovar, J. Strohalm, P. Chytil, B. Rihova, K. Ulbrich, J. Control. Release 2011, 154, 241; b) S. Aryal, C. M. J. Hu, L. F. Zhang, ACS Nano 2010, 4, 251; c) Y. Bae, S. Fukushima, A. Harada, K. Kataoka, Angew. Chem.-Int. Edit. 2003, 42, 4640; d) Y. M. Zhao, et al., Nat. Commun. 2016, 7.

[21] a) F. Danhier, O. Feron, V. Preat, J. Control. Release 2010, 148, 135; b) M. M. Chen, et al., Oncotarget 2017, 8, 45759.

[22] a) G. Sahay, D. Y. Alakhova, A. V. Kabanov, J. Control. Release 2010, 145, 182; b) S. Mukherjee, R. N. Ghosh, F. R. Maxfield, Physiol. Rev. 1997, 77, 759.

[23] a) J. O. Eloy, M. C. de Souza, R. Petrilli, J. P. A. Barcellos, R. J. Lee, J. M. Marchetti, Colloid Surf. BBiointerfaces 2014, 123, 345; b) V. P. Torchilin, Nat. Rev. Drug Discov. 2005, 4, 145.

[24] a) D. J. McClements, Soft Matter 2012, 8, 1719; b) N. Anton, T. F. Vandamme, Pharm. Res. 2011, 28, 978; c) L. Salvia-Trujillo, R. Soliva-Fortuny, M. A. Rojas-Grau, D. J. McClements, O. Martin-Belloso, in Annu. Rev. Food. Sci. Technol., Vol. 8 (Eds.: M. P. Doyle, T. R. Klaenhammer), Annual Reviews, Palo Alto, 2017, pp. 439; d) N. Anton, J. P. Benoit, P. Saulnier, J. Control. Release 2008, 128, 185; e) A. S. Klymchenko, F. Liu, M. Collot, N. Anton, Advanced Healthcare Materials 2020, DOI: 10.1002/adhm.202001289.

[25] R. Bouchaala, L. Mercier, B. Andreiuk, Y. Mely, T. Vandamme, N. Anton, J. G. Goetz, A. S. Klymchenko, J. Control. Release 2016, 236, 57.

[26] E. Roger, J. C. Gimel, C. Bensley, A. S. Klymchenko, J. P. Benoit, J. Control. Release 2017, 253, 11.

[27] A. S. Klymchenko, E. Roger, N. Anton, H. Anton, I. Shulov, J. Vermot, Y. Mely, T. F. Vandamme, RSC Adv. 2012, 2, 11876.

[28] A. L. D. Camara, G. Nagel, H. R. Tschiche, C. M. Cardador, L. A. Muehlmann, D. M. de Oliveira, P. Q. Alvim, R. B. Azevedo, M. Calderon, J. P. F. Longo, Nanomedicine 2017, 12, 1751.

[29] a) R. B. Ibrahim, C. Liu, S. M. Cronin, B. C. Murphy, R. Cha, P. Swerdlow, D. J. Edwards, Pharmacotherapy: The Journal of Human Pharmacology and Drug Therapy 2007, 27, 1529; b) C. W. Cheng, J. E. Hendrickson, C. A. Tormey, D. Sidhu, American Journal of Clinical Pathology 2017, 148, 190; c) J. T. Kielstein, A. Schwarz, A. Arnavaz, O. Sehlberg, H. M. Emrich, D. Fliser, Clin. Nephrol. 2002, 57, 484. 
[30] a) C. C. Lee, E. R. Gillies, M. E. Fox, S. J. Guillaudeu, J. M. J. Frechet, E. E. Dy, F. C. Szoka, Proc. Natl. Acad. Sci. U. S. A. 2006, 103, 16649; b) X. P. Duan, J. S. Xiao, Q. Yin, Z. W. Zhang, H. J. Yu, S. R. Mao, Y. P. Li, ACS Nano 2013, 7, 5858; c) X. Q. Yang, J. J. Grailer, I. J. Rowland, A. Javadi, S. A. Hurley, V. Z. Matson, D. A. Steeber, S. Q. Gong, ACS Nano 2010, 4, 6805.

[31] a) K. E. Sapsford, L. Berti, I. L. Medintz, Angew. Chem.-Int. Edit. 2006, 45, 4562; b) B. L. SanchezGaytan, F. Fay, S. Hak, A. Alaarg, Z. A. Fayad, C. Perez-Medina, W. J. M. Mulder, Y. Zhao, Angew. Chem.-Int. Edit. 2017, 56, 2923.

[32] L. Bromberg, M. Temchenko, T. A. Hatton, Langmuir 2002, 18, 4944.

[33] G. Nagel, H. R. Tschiche, S. Wedepohl, M. Calderon, J. Control. Release 2018, 285, 200.

[34] a) C. Simonsson, G. Bastiat, M. Pitorre, A. S. Klymchenko, J. Bejaud, Y. Mely, J. P. Benoit, Eur. J. Pharm. Biopharm. 2016, 98, 47; b) A. Saito, S. Yamamoto, R. Ochi, K. Inoue, S. Hadano, S. Watanabe, T. Nakayama, Y. Niko, Bull. Chem. Soc. Jpn. 2020, 93, 568.

R. Crespo, M. Pizarro, Avian Dis. 2006, 50, 309.

\section{Table of Contents}

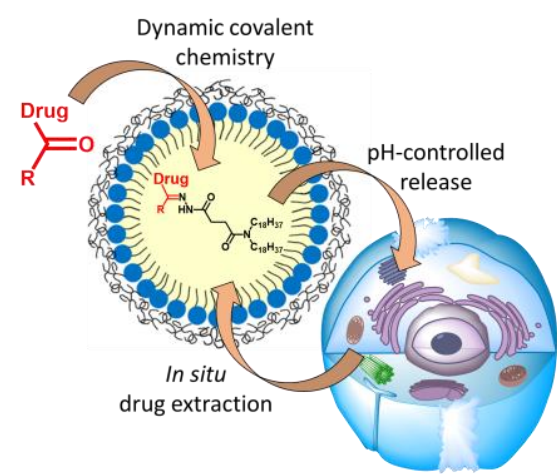

Drug-sponge nanocarrier that reversibly captures and releases drugs or contrast agents under $\mathrm{pH}$ control is developed using dynamic covalent chemistry. Inside this lipid droplet nano-reactor, lipophilic capture molecules transform free cargo-ketones into encapsulated hydrazone prodrugs, enabling controlled release of active cargos and their extraction from cells and tissues for detoxification. 